\title{
propuesta de metodología para la estimación de la resistencia del hormigón en un punto de una estructura a través de ensayos de información, en éste $y / u$ otros puntos
}

CARLOS BUXADE RIBOT y JUAN MARGARIT CONSARNAU, Dres, arquitectos

sinopsis El carácter destructivo de la extracción de probetas testigo, actualmente el único ensayo de información que facilita datos de partida fidedignos desde el punto de vista resistente, obliga a asignar una resistencia a zonas de la estructura más o menos lejanas de los puntos de extracción.

Las variables del problema son el número de extracciones y su localización. En un extremo tendríamos el caso de la obtención de testigos en todas las piezas estructurales, con el consiguiente riesgo de debilitación de la estructura, sobre todo durante las operaciones necesarias para ello, y el gravoso factor económico y de tiempo que este planteamiento lleva aparejado. En el otro extremo se situaría el caso de la evaluación de la resistencia de una gran zona de estructura en base a los resultados de la rotura de un mínimo número de probetas, a través de una extrapolación sin las necesarias garantías.

Naturalmente, el número de ensayos de información y su localización sitúa el problema en una región intermedia entre los dos extremos expuestos. La necesaria extrapolación de unos resultados, forzosamente no exhaustivos, se realiza, entonces, con una mayor o menor garantía en función del conocimiento previo de la ejecución de la obra: cuanto mayor es este conocimiento más fácilmente se podrá establecer la relación de resistencias entre los testigos y las piezas que, sin tener probetas que los representen directamente, puedan, por proximidad o ritmo de ejecución conocido, asimilarse a las características de dichos testigos.

El problema más grave se plantea cuando el desconocimiento de la ejecución de la obra no permite establecer nexos de conocimiento entre los resultados obtenidos en determinados testigos y el resto de la zona de estructura cuya capacidad resistente se trata de evaluar.

En este caso no parece posible el establecimiento de relaciones entre dos puntos de la estructura más que basándose en las distancias físicas y los resultados obtenidos en los testigos ensayados. Este trabajo trata de la aplicación de la teoría de la información para cuantificar estas relaciones y agrupar los puntos en los cuales el hormigón es objeto de estimación resistente.

\section{HIPOTESIS DE PARTIDA}

En primer lugar, supondremos que la zona de estructura cuya evaluación resistente se trata de efectuar tiene un carácter homogéneo en cuanto a su proceso general de hormigonado e hipotética distribución de «lotes» de material. Se tratará, pues, por ejemplo, de un forjado o de los pilares de una planta, ya que se puede afirmar, razonablemente, que no se habrán hormigonado unos pocos pilares, después su porción de forjado, más adelante otros pilares, etc. 
Es decir, el carácter homogéneo vendrá dado por la tipología de las piezas y su vecindad básicamente: pilares en un caso y forjados en otro y agrupados por piezas, en los casos más corrientes.

\section{DEFINICION DE VARIABLES Y LAZOS}

Supondremos, entonces, quedada una zona «homogénea» de estructura a evaluar, se han fijado una serie de puntos $P_{1}, P_{2}, \ldots, P_{n}$, en cada uno de los cuales debe asignarse una resistencia al hormigón, y consideraremos estas $n$ resistencias como variables aleatorias binarias en el sentido de que pueden ser conocidas o pueden no ser conocidas.

La relación entre dos puntos $i$, $j$, vendrá medida por la intensidad del lazo $l_{i j}$ que los une, intensidad que debe basarse en nuestro previo conocimiento sobre $i$ y $j$. Como primera aproximación podría definirse una componente de situación, que llamaremos «grado de afinidad topológica entre $i$ y $j_{\text {» }}\left(t_{i j}\right)$, y una componente resistente, que llamaremos «grado de afinidad resistente entre $i$ y $j$ » $\left(r_{i j}\right)$. Si suponemos que idéntico "peso» debe tener en nuestro conocimiento el hecho de que los puntos $i$ y $j$ estén muy próximos o muy lejanos (afinidad topológica), o el hecho de que, si extrajéramos probetas testigo en ambos puntos, las resistencias de ambas muestras fueran muy semejantes o muy dispares (afinidad resistente), podemos asignar valores entre 0 y 5 al grado de afinidad resistente y lo mismo al grado de afinidad topológica, para así obtener un lazo $l_{i j}$ cuya intensidad total quedara enmarcada entre 0 y 10 , como valores mínimo y máximo.

\subsection{Definición del grado de afinidad topológica}

\subsubsection{Forjados}

Llamando $S_{0}$ a la superficie que puede suponerse asignada a un «lote» en el momento del hormigonado $\left(500 \mathrm{~m}^{2}\right.$, por ejemplo) y $d_{i j}$ a la distancia entre el punto $i$ y el punto $j$ :

$$
\begin{array}{ll}
\text { si } d_{i j} \leqslant \sqrt{S_{0}} & t_{i j}=\frac{\sqrt{S_{0}}-d_{i j}}{\sqrt{S_{0}}}+4 \\
\text { si } d_{i j}>\sqrt{S_{0}} & t_{i j}=\frac{\left(1,5 \cdot \sqrt{S_{0}}-d_{i j}\right) \cdot 4}{0,5 \cdot \sqrt{S_{0}}}\left(\text { si } t_{i j}<0, \text { tomar } t_{i j}=0\right) .
\end{array}
$$

\subsubsection{Pilares}

Llamando $S$ a la superficie de la zona homogénea de estructura cuya resistencia debemos evaluar en $n$ puntos, $n_{p}$ al número de pilares que existen en dicha zona, $n_{0}$ al número máximo de pilares que pueden suponerse formando parte de un mismo lote (25, por ejemplo) y $d_{i j}$ la distancia entre el punto $i$ y el punto $j$ :

$$
\begin{aligned}
& \text { si } d_{i j} \leqslant\left(\sqrt{n_{0}}-1\right) \cdot \sqrt{\frac{S}{n_{p}}} t_{i j}=\frac{\left[\left(\sqrt{n_{0}}-1\right) \cdot \sqrt{\frac{S}{n_{p}}}\right]-d_{i j}}{\left[\left(\sqrt{n_{0}}-1\right) \cdot \sqrt{\frac{S}{n_{p}}}\right]}+4 \\
& \text { si } d_{i j}>\left(\sqrt{n_{0}}-1\right) \cdot \sqrt{\frac{S}{n_{p}}} \quad t_{i j}=\frac{\left[1,5 \cdot\left(\sqrt{n_{0}}-1\right) \cdot \sqrt{\frac{S}{n_{p}}}\right]-d_{i j}}{0,5 \cdot\left(\sqrt{n_{0}}-1\right) \cdot \sqrt{\frac{S}{n_{p}}} \cdot 4} \\
& \quad\left(\operatorname{si~} t_{i j}<0, \text { tomar } t_{i j}=0\right) .
\end{aligned}
$$


NOTA:

La fijación exacta de los valores anteriores no pretende ser el objetivo de este estudio, cuya finalidad quiere ser, sólo, la de señalar una posible dirección de trabajo, en base a la teoría de la información. Es con vistas a una ejemplificación que seguirá y que puede aclarar al lector el contenido de lo expuesto, que se han definido las afinidades topológicas y que se definirán las resistentes. Si el camino general emprendido parece correcto, su virtud consistirá, precisamente, en que la atención y modificaciones podrán centrarse en aspectos muy concretos, como los de los grados de afinidad, obteniéndose así, al menos, un descenso en el grado de complejidad del problema primitivo.

Las expresiones [1], [2], [3] y [4] se han obtenido imponiendo un valor $t_{i j}=4$ cuando $d_{i j}=\alpha$,

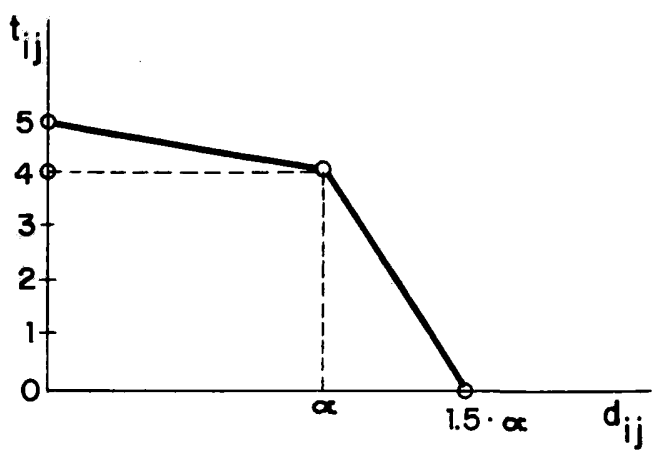
$t_{i j}=0$ si $d_{i j} \geqslant 1,5 \cdot \alpha$ y $t_{i j}=5$ si $d_{i j}=0$, con variación lineal entre los tres puntos.

El valor de $\alpha$ es $\sqrt{S_{0}}$ en el caso de forjados y

$$
\left(\sqrt{n_{0}}-1\right) \cdot \sqrt{\frac{S}{n_{p}}}
$$

en el caso de pilares.

\subsection{Definición del grado de afinidad resistente}

Entre un punto $i$ y un punto $j$ se establecen las siguientes expresiones para determinar el grado de afinidad resistente:

2.2.1. En el punto i se conoce la resistencia del hormigón a través de un ensayo de información. En el punto $j$ no se tiene ensayo de información

$$
r_{i j}=\left(1-\frac{f_{c, i}-f_{c, \min }}{f_{c, \max }-f_{c, \min }}\right) \cdot 5 \quad\left(\text { si } r_{i j}<0, \text { tomar } r_{i j}=0\right) .
$$

Siendo:

$f_{c, i}=$ resistencia obtenida en el punto $i$ a través del ensayo de información.

$f_{c, \min }=$ la menor de las resistencias obtenidas mediante ensayos de información en la zona homogénea objeto de estudio.

$f_{c, \max }=$ la mayor de las resistencias obtenidas mediante ensayos de información en la zona homogénea objeto de estudio.

2.2.2. En los puntos $i$ y $j$ se conoce la resistencia del hormigón a través de ensayos de información

$$
r_{i, j}=\left(1-\frac{f_{M}-f_{m}}{f_{m}}\right) \cdot 5 \quad\left(\text { si } r_{i j}<0, \text { tomar } r_{i j}=0\right) .
$$

Siendo:

$f_{M}$ y $f_{m}=$ la mayor y la menor de las resistencias $f_{c, i}$ y $f_{c, i}$ obtenidas mediante ensayos de información en los puntos $i$ y $j$. 
2.2.3. En los puntos $i$ y j no se conoce la resistencia del hormigón

$$
r_{i j}=0
$$

\subsection{Intensidad del lazo entre el punto $i$ y el punto $j$}

$$
l_{i j}=t_{i j}+r_{i j}
$$

Para la correcta aplicación de las expresiones de teoría de la información del apartado 3, es preciso que $l_{i j}$ tenga valores enteros. Se prescindirá de los decimales si éstos no superan el valor 0,5 , y se le asignará el valor del entero superior cuando los decimales sean mayores que 0,5.

\section{INFORMAGION PERDIDA AL TRATAR, CONJUNTAMENTE O POR PARTES, LOS $n$ PUN* TOS EN LOS QUE SE PRETENDE ESTIMAR LA RESISTENGIA DEL HORMIGON}

Se tiene, pues, definido un sistema de $n$ puntos relacionados entre sí por lazos de intensidad discontinua y variable entre 0 y 10.

Si se tratan los $n$ puntos simultáneamente la información perdida por el sistema es, evidentemente, nula. Pero, en este caso, la resistencia en los puntos sin testigo habrá de relacionarse con el mínimo valor de la resistencia obtenida en los puntos con ensayo de información. De ahí que sea conveniente, en general, agrupar los $n$ puntos en distintos subsistemas, tales que su conjunto sea una partición del sistema total, para así intentar delimitar en un mismo subsistema los puntos bajo la influencia de los testigos de menores resistencias y no disminuir así la resistencia en los restantes puntos.

Cada subsistema debe contener, al menos, un punto en el cual se ha obtenido la resistencia a través de un ensayo de información.

Este proceso lleva, entonces, implícita una pérdida de información que debe incidir en la capacidad resistente asignada al hormigón en los $n$ puntos del sistema.

\subsection{Información perdida al considerar una par- tición $\pi$ cualquiera del sistema de $n$ puntos}

La información perdida al considerar los subsistemas $S_{1}, S_{2}, \ldots, S_{k}$ (partición $\pi$ ) en lugar del sistema total $S$ puede asimilarse al valor de la expresión (*):

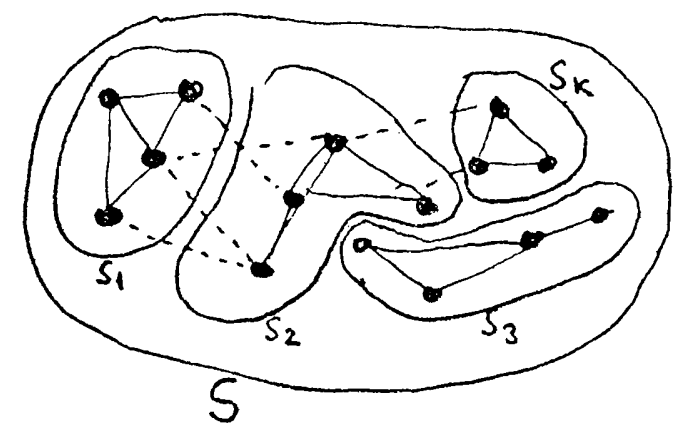

$$
R(\pi)=\frac{\frac{10 \cdot n \cdot(10 \cdot n-1)}{2} \cdot \sum_{\pi} \nu_{i j}-(L+45 \cdot n) \cdot 100 \cdot \sum_{\pi} S_{\alpha} \cdot S_{\beta}}{\sqrt{100 \cdot \sum_{\pi} S_{\alpha} \cdot S_{F} \cdot\left(\frac{10 \cdot n \cdot(10 \cdot n-1)}{2}-100 \cdot \sum_{\pi} S_{\alpha} \cdot S_{\beta}\right)}}
$$

(*) C. BuXadé-J. Margarit: Introducción a una teoría del Conocimiento de la Arquitectura y del Diseño. Ed. Blume, 1969. 
Siendo:

$n=$ número de puntos del sistema $S$ (o sea, número de puntos en los cuales tratamos de estimar la resistencia del hormigón).

$L=\sum l_{i j}=$ suma de todos los lazos $l_{i j}$ del sistema $S$.

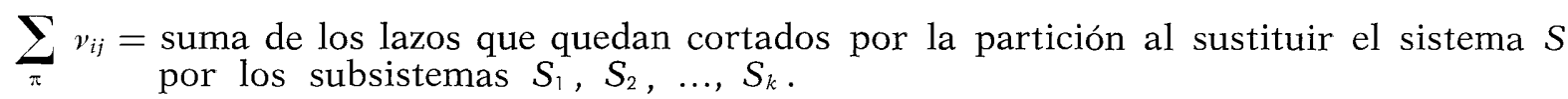

$\sum S_{\alpha} \cdot S_{\beta}=$ suma de todos los productos posibles de los dos números que representan el número de puntos $S_{\alpha}$ y $S_{\beta}$ de dos subsistemas de la partición $\pi$.

Por ejemplo: si $S_{1}=7 ; S_{2}=4$, y $S_{3}=5$, es:

$\sum_{\pi} S_{x} \cdot S_{\beta}=7 \times 4+7 \times 5+4 \times 5$

\subsection{Partición óptima}

Será, evidentemente, la que, de todas las posibles, dé un valor mínimo a la expresión [8] de $R(\pi)$. Debido a la discontinuidad de la función $R(\pi)$ este problema no tiene más solución que el tanteo combinatorio de las distintas posibilidades.

Un programa para ordenador electrónico que resuelve el problema se expone en la obra anteriormente citada.

Admitiremos como valor mínimo de la información perdida, por debajo del cual esta pérdida de información no penalizará la resistencia del hormigón, el que podemos asimilar a la siguiente expresión:

$$
R^{0}(\pi)=\frac{\frac{10 \cdot n \cdot(10 \cdot n-1)}{2} \cdot \frac{T}{n} \cdot \frac{n_{p} \cdot\left(n_{p}-1\right)}{2} \cdot\left(\frac{n}{n_{p}}\right)-(L+45 \cdot n) \cdot 25 \cdot n^{2}}{\sqrt{25 \cdot n^{2} \cdot\left(\frac{10 \cdot n \cdot(10 \cdot n-1)}{2}-25 \cdot n^{2}\right)}}
$$

Siendo:

$$
n_{p}=\text { número de subsistemas en que queda dividido el sistema } S \text { después de la partición. }
$$

$T=\sum t_{i j}=$ suma de los grados de afinidad topológica $t_{i j}$ del sistema $S$.

Esta mínima información perdida admitida correspondería a la información perdida por el sistema $S$ al efectuar una partición en $n_{p}$ subsistemas del mismo número de elementos $\left(n / n_{p}\right)$ y considerar que todos los puntos están relacionados entre sí con lazos de valor la media aritmética de los lazos topológicos del sistema $S$ que se estudia.

\subsection{Partición pésima}

Será aquella que acarree una pérdida máxima de información al sistema. Admitiremos, para este caso, como valor máximo de la información perdida, a partir del cual no es posible asignar ningún valor a la resistencia del hormigón en la estructura considerada, el que podemos asimilar a la siguiente expresión:

$$
R^{p}(\pi)=\frac{\frac{10 \cdot n \cdot(10 \cdot n-1)}{2} \cdot(L+45 \cdot n)-(L+45 \cdot n) \cdot(n-2) \cdot[50 \cdot(n-3)+200]}{\sqrt{(n-2)[50 \cdot(n-3)+200] \cdot\left(\frac{10 \cdot n \cdot(10 \cdot n-1)}{2}-(n-2) \cdot[50 \cdot(n-3)+200]\right)}}[
$$


Esta máxima información perdida admitida correspondería a la información perdida por el sistema $S$ con sus lazos $l_{i j}$ reales, al efectuar una partición en $(n-1)$ subsistemas de un elemento en cada subsistema, a excepción de un subsistema con dos elementos que se suponen no relacionados entre sí.

\section{NOTA:}

No se admitirán, naturalmente, particiones que agrupen en un mismo subsistema elementos separados por otros elementos de otros subsistemas.

\subsection{Penalización de la resistencia obtenida mediante ensayos de información en función de la información perdida}

Se considerará como resistencia estimada del hormigón en los puntos $i$ del sistema $S$ en los que se han efectuado ensayos de información con resistencias $f_{c, i}$ el valor $f_{c, i}$ :

$$
f_{e, i}=f_{c, i} \cdot \frac{R(\pi)-R^{P}(\pi)}{R^{0}(\pi)-R^{P}(\pi)}
$$

Si $R(\pi)>R^{0}(\pi)$, tomar $R(\pi)=R^{0}(\pi)$.

Si no se efectúa partición, y por tanto, se considera a la vez todo el sistema $S$, deberá tomarse $R(\pi)=R^{0}(\pi)$. Esta solución es la correcta cuando la zona homogénea a estudiar es pequeña (de superficie inferior a la normal de un lote) o cuando se conoce la resistencia del hormigón, mediante ensayos de información, en los $n$ puntos del sistema.

\subsection{Estimación de la resistencia del hormigón en los puntos $j$ en los cuales no se han efectuado} ensayos de información

$$
f_{e, j}=0,2 \cdot t_{j, K} \cdot f_{e, \min }
$$

Siendo:

$K=$ punto del mismo subsistema que el punto $j$ y que cumple las dos condiciones siguientes:

1. $\quad$ En él se ha efectuado ensayo de información.

2. ${ }^{a} \quad$ De todos los puntos con ensayo de información del mismo subsistema que el punto $j, K$ es el más próximo al $j$.

$t_{j, K}=$ valor del grado de afinidad topológica entre $j$ y $K$.

$f_{e, \min }=$ de todas las resistencias $f_{e, i}$ estimadas en los puntos con ensayo de información pertenecientes al mismo subsistema que el punto $j$ (3.4), la de valor más bajo.

\subsection{Resistencia estimada en cada punto}

Si se han efectuado distintas particiones $\pi_{1}, \pi_{2}, \ldots, \pi_{m}$ para cada una de las cuales la resistencia estimada en un punto $i$ ha sido de $f_{e, i}\left(\pi_{1}\right), f_{e, i}\left(\pi_{2}\right), \ldots, f_{e, i}\left(\pi_{m}\right)$, se tomará el máximo valor como resistencia estimada en dicho punto. 


\section{EJEMPLO DE APLICACION}

Estimar la resistencia del hormigón en los diez pilares de la zona de estructura que a continuación se indica, habiéndose efectuado ensayos de información en los soportes 4, 6, 8 y 9 con resultados de $170,100,150$ y $160 \mathrm{kp} / \mathrm{cm}^{2}$, respectivamente.

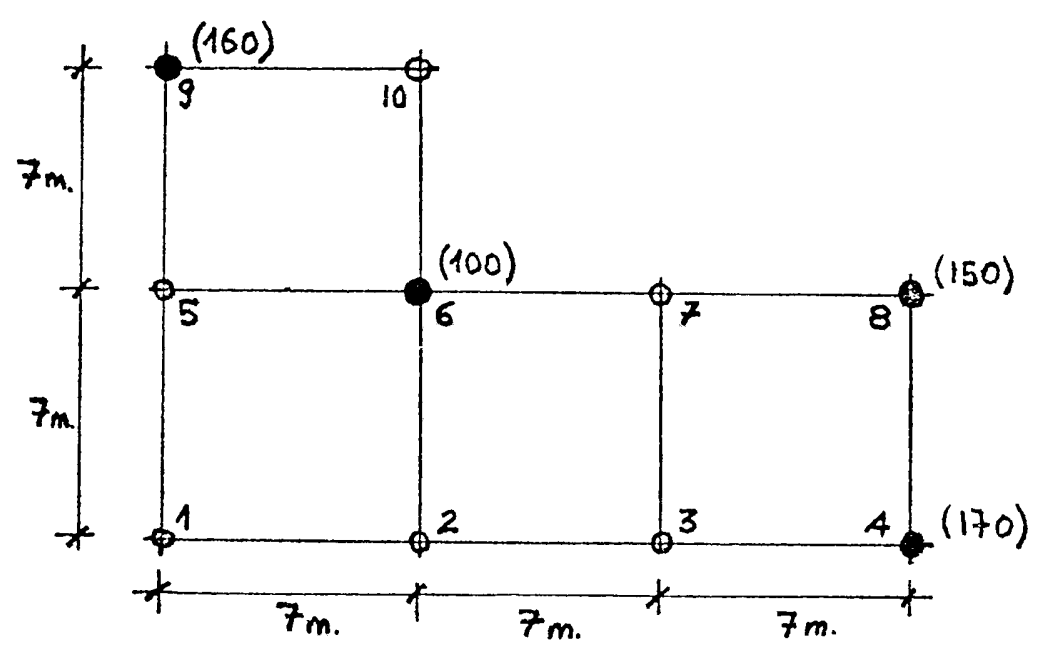

Lazos del sistema:

\begin{tabular}{|c|c|c|c|c|c|c|c|c|c|c|}
\hline & 10 & 9 & 8 & 7 & 6 & 5 & 4 & 3 & 2 & \\
\hline \multirow{3}{*}{1} & 4,1 & 4,2 & 2 & 4,1 & 4,4 & 4,6 & 2,5 & 4,2 & 4,6 & $t$ \\
\hline & 0 & 0,71 & 1,42 & 0 & 5 & 0 & 0 & 0 & 0 & $r$ \\
\hline & 4 & 5 & 3 & 4 & 9 & 5 & 2 & 4 & 5 & 1 \\
\hline \multirow{3}{*}{2} & 4,2 & 4,1 & 4,1 & 4,4 & 4,6 & 4,4 & 4,2 & 4,6 & $t$ & \\
\hline & 0 & 0,71 & 1,42 & 0 & 5 & 0 & 0 & 0 & $r$ & \\
\hline & 4 & 5 & 6 & 4 & 10 & 4 & 4 & 5 & 1 & \\
\hline \multirow{3}{*}{3} & 4,1 & 3 & 4,4 & 4,6 & 4,4 & 4,1 & 4,6 & $t$ & & \\
\hline & 0 & 0,71 & 1,42 & 0 & 5 & 0 & 0 & $r$ & & \\
\hline & 4 & 4 & 6 & 5 & 9 & 4 & 5 & 1 & & \\
\hline \multirow{3}{*}{4} & 3 & 0,6 & 4,6 & 4,4 & 4,1 & 2 & $t$ & & & \\
\hline & 0 & 4,69 & 4,33 & 0 & 1,5 & 0 & $r$ & & & \\
\hline & 3 & 5 & 9 & 4 & 6 & 2 & $I$ & & & \\
\hline \multirow{3}{*}{5} & 4,4 & 4,6 & 2,5 & 4,2 & 4,6 & $t$ & & & & \\
\hline & 0 & 0,71 & 1,42 & 0 & 5 & $r$ & & & & \\
\hline & 4 & 5 & 4 & 4 & 10 & 1 & & & & \\
\hline \multirow{3}{*}{6} & 4,6 & 4,4 & 4,2 & 4,6 & t & & & & & \\
\hline & 5 & 2 & 2,5 & 0 & $r$ & & & & & \\
\hline & 10 & 6 & 7 & 5 & 1 & & & & & \\
\hline \multirow{3}{*}{7} & 4,4 & 4,3 & 4,6 & $t$ & & & & & & \\
\hline & 0 & 0,71 & 1,42 & $r$ & & & & & & \\
\hline & 4 & 5 & 6 & 1 & & & & & & \\
\hline \multirow{3}{*}{8} & 4,1 & 2 & $t$ & & & & & & & \\
\hline & 1,42 & 4,66 & $r$ & & & & & & & \\
\hline & 6 & & 1 & & & & & & & \\
\hline \multirow{3}{*}{9} & 4,6 & $t$ & & & & & & & & \\
\hline & 0,72 & $r$ & & & & & & & & \\
\hline & 5 & 1 & & & & & & & & \\
\hline
\end{tabular}


$L=237$.

$R^{0}(\pi)=-493$ (partición en dos subsistemas).

$R^{0}(\pi)=-702$ (partición en tres subsistemas).

$R^{p}(\pi)=+243$.

\begin{tabular}{|c|c|c|c|c|c|c|c|}
\hline PALORES & $\frac{1,2,5,6,9,20}{3,4,7,8}$ & $\frac{1,2,3,4}{5,6,7,8,9,20}$ & $\frac{4,8}{1,2,3,5,6,7,9,29}$ & $\frac{6}{1,2,3,4,5,7,8,9,19}$ & $2,3,9$ & $\begin{array}{c}1,2,3,4,5,6 \\
7,3,9,10\end{array}$ & \\
\hline & & & & & 4,8 & & \\
\hline$\sum \nu_{i j}$ & 111 & 124 & 76 & 72 & 153 & 0 & \\
\hline$R(\pi)$ & -444 & -418 & -312 & -237 & -573 & -493 & \\
\hline$\frac{R(\pi)-R^{p}(\pi)}{R^{0}(\pi)-R^{p}(\pi)}$ & 0,93 & 0,90 & 0,75 & 0,52 & 0.86 & 1 & $\begin{array}{l}\text { RESISTEN } \\
\text { CIA ESTI } \\
\text { MADA }\end{array}$ \\
\hline $1_{e, 4}$ & 158 & 253 & 127,5 & 88.4 & 146,2 & 170 & 170 \\
\hline $\mathrm{f}_{\mathrm{e}, 6}$ & 93 & 90 & 75 & 52 & 86 & 100 & 100 \\
\hline $\mathrm{r}_{\mathrm{e}, 8}$ & 139.5 & 135 & 212,5 & 78 & 129 & 150 & 150 \\
\hline$t_{0,9}$ & 248,8 & 244 & 220 & 83,2 & 237,6 & 160 & 260 \\
\hline$r_{e, 1}$ & 81,84 & 76,5 & 66 & 65,52 & 215,58 & 88 & 115.58 \\
\hline $\mathrm{r}_{\mathrm{e}, 2}$ & 85,56 & 128,52 & 69 & 65,52 & 79,12 & 92 & 128,52 \\
\hline$f_{e, 3}$ & 122,76 & 140,76 & 66 & 72,76 & 75,68 & 92 & 140,76 \\
\hline$f_{e, 5}$ & 85,56 & 82,8 & 69 & 71,76 & 126,59 & 92 & 126.59 \\
\hline$f_{e, 7}$ & 128,34 & 82,8 & 69 & 71,76 & 79,12 & 92 & 128,34 \\
\hline $1_{e, 10}$ & 85,56 & 82,8 & 69 & 71,76 & 79,12 & 92 & 92 \\
\hline
\end{tabular}

\section{CONSIDERAGIONES SOBRE EL METODO PROPUESTO}

El objetivo de la metodología aquí propuesta no es el de «acertar» la real resistencia del hormigón en los puntos donde no se dispone de ensayos de información, tarea digna de magos y adivinos, sino el intento de asignar una resistencia razonablemente segura a todos los puntos considerados globalmente, de forma que los «números* resultantes miden, más que una serie independiente de resistencias, nuestro conocimiento de la zona de estructura estudiada.

Este conocimiento ha de depender forzosamente de la forma en que se ha obtenido; de ahí que, según la partición escogida, varíe el resultado, ya que este resultado viene afectado por lo que podríamos llamar un coeficiente de desconocimiento, una medida de nuestra ignorancia de las características del material.

Partiendo, pues, de los únicos datos objetivos que se poseen, unos ensayos de información en algunos puntos y las distancias de los puntos entre sí, el método pondera el mayor o menor número de probetas testigo, la mayor o menor superficie a estudiar partiendo de este número de ensayos, la proximidad o alejamiento de cada punto (cuya resistencia se desconoce totalmente) a alguno de los testigos, la mayor o menor homogoneidad de los subsistemas de puntos a considerar conjuntamente penaliza la «astucia» de los intentos de aislamiento de las probetas testigo con resultados más bajos, etc., etc.

Mide, en resumen, la cantidad de información que se pierde al contemplar de una determinada manera la estructura y obtiene los resultados en función de esta información perdida. 


\section{résumé}

Proposition de méthodologie pour l'estimation de la résistance du béton sur un point d'une structure moyennant des essais d'information, sur ce point et/ou d'autres points

Carlos Buxadé Ribot et Juan Margarit Consarnau, Dr. architectes

Le caractère destructif du prélèvement de carottes, actuellement le seul essai d'information qui fournit des données de départ dignes de foi du point de vue résistance, oblige à assigner une résistance à des zones de la structure plus ou moins éloignées des points du prélèvement.

Les variables du problème sont le nombre de prélèvements et leur localisation. D'une part, nous aurions le cas du prélèvement de carottes de toutes les pièces structurales avec le risque d'affaiblissement de la structure, surtout durant les opérations de prélèvement, et l'onéreux facteur économique
et de temps que cette opération implique. D'autre part, il serait le cas de l'évaluation de la résistance d'une grande zone de structure sur la base des résultats de la rupture d'un nombre minimum d'éprouvettes, par une extrapolation sans les garanties nécessaires.

Evidemment, le nombre d'essais d'information et leur localisation situe le problème dans une région intermédiaire entre les deux cas mentionnés. L'extrapolation nécessaire de quelques résultats, forcément non exhaustifs, se réalise alors avec plus ou moins de garantie en fonction de la connaissance préalable de l'exécution des travaux: plus cette connaissance est grande, plus facilement on pourra établir le rapport de résistances entre les carottes et les pièces qui, sans avoir d'éprouvettes qui les représentent directement, pouissent, pour proximité au rythme d'éxécution connu, s'assimi. ler aux caractéristiques de ces carottes.

Le problème le plus grave se pose Iorsque la méconnaissance de l'exécution de l'ouvrage ne permet pas d'établir des rapports de connaissances entre les résultats obtenus sur certaines carottes et le reste de la zone de structure dont il est question d'évaluer la capacité de résistance.

Dans ce cas il ne semble possible d'établir des rapports entre deux points de la structure que sur la base des distances physíques et des résultats obtenus sur les carottes essayées. Ce travail traite de l'application de la théorie de l'information pour quantifier ces rapports et grouper les points sur lesquels le béton est l'objet d'estimation résistante.

\section{summary}

Proposal for Method for Evaluating Concrete Strength at a Place in a Structure by means of Information Tests, at this and/or other Points

Carlos Buxadé Ribot and Juan Margarit Consarnau, Dr. Architects

The destructive character of the extraction of test specimens, which at present is the only test that provides trustworthy data with regard to strength, makes it necessary to assign a strength to areas in the structure that are at a greater or lesser distance from the extraction points.

The variables of the problem are the number of extractions and their emplacement. At one end we would have the case in which samples are taken from all the structural parts at the risk of weakening the structure, above all during the sampling process itself, as well as the negative financial and time factors, that this procedure would imply. At the other far end we would place the case of evaluating the strength in a great
structural area on the basis of the results from breaking a small quantity of test pieces, by way of an extrapolation without the required guarantees.

Naturally, the number of test and their emplacement situates the problem in between the two above mentioned extremes. The necessary extrapolation of some results that by nature are not exhaustive, is then carried out with greater or lesser guarantee with regard to advance knowledge of the which without being represented directly by samples, can adapt themselves to their characteristics, either by proximity or known execution rhythm.

The most important problem arises when there is not enough knowledge on how the work was carried out, so that it is impossible to relate the strength of the test specimens with the actual strength of the rest of the structure under investigation.

In this case it does not seem possible to establish the relation between two points in the structure other than on the basis of the actual physical distances and the results obtained through the test specimens. This report dealts with the application of the information theory to quantify these relations and to group the points at which the concrete strength is estimated.

\section{zusammenfassung}

Methodologievorschlag fur das Schatzen der Betonfestigkeit an einer Stelle des Baus mittelst Informationsproben, an dieser und/oder anderen Stellen

Carlos Buxadé Ribot und Juan Margarit Consarnau, Dr. Architekten

Die zerstörende Beschaffenheit der Probeentnahme, welches zur Zeit das ainzige Verfahren ist, das zuverlässĩge Daten in Bezug auf Festigkeit darbindet, macht es esforderlich, den den Entnahmestellen mehr oder weniger entfernten Gebieten des Baukörprs eine Trägfähígkeit zuzuteilen.

Die Veränderlichen des Problems sind die Entnahmezahl und die Entnahmestelle. An dem einen Ende hätten wir den Fall, in dem allen Baugliedern Proben entnommen werden, mit der dadurch entstehenden Schwächungsgefahr des Baus, vor allem gerade während der Probenahme, wie auch den negativen ökonomischen und zeitlichen Faktoren, die dieses Verfahren mit sich bringt. An dem anderen Ende hätten wir den Fall, in dem die Festig keit eines grossen Baukörpergebietes auf Grund der Bruchergebnisse einer geringen Zahl von Probestücken und Extrapolieren ohne erforderliche Garan
tie geschätzt wird.

Die Probezahl und die Entnahmestelle versetzen das Problem in die Mitte der beiden erwähnten Punkte. Das erforderliche Extrapolieren einiger, durch je grösser diese Kenntnis ist, umso leichter ist es, das Verhältnis zwischen den Probestücken und den Baugliedern festzustellen. Diese letzteren je grösser diese Kenntnis ist, umso leichter ist es, das Verhältnis zwischen den Probestücken und den Baugliedern festzustellen. Diese letzteren
sind nicht direkt durch Probestücke repräsentiert aber können sich an die Eingenschaften dieser Probestücke entweder durch Nähe oder gekannte Ausführungstakt angleichen.

Das grösste Problem entsteht, wenn nicht genug Kenntnis vorhanden ist, wie der Bau ausgeführt worden ist, wodurch es nicht möglich ist, die Festigkeit der Probestücke mit der des übrigen Gebietes des Baukörpers, zu vergleíchen.

In diesem Falle scheint es nur möglich zu sein, das Verhältnis zwischen zwei Punkten des Baus auf Grund des physischen Abstandes und der in den untersuchten Probestücken erhaltenen Ergebnisse festzustellen. Diese Arbeit behandelt das Verwenden disr Informationstheorie, um diese Verhältnisse zu quantifieren, und die Stellen, an denen die Festigkeit des Betons geschätzt wird, zu gruppieren. 